Disposition To Pay For Bear Fruit Characteristic: A Symbiotic Investigation

R.H.M.

Department Of Agricultural Economics, Arak University ,Iran

\begin{abstract}
Purpose: Identification of consumer preferences is important in designing products. Producers will immensely benefit by knowing them to capture the market share. In light of this, we evaluate consumers' stated preferences for various Characteristic of four popular Bear Fruits: grapes, sweet orange, pear and pomegranate. Research
\end{abstract}

\title{
Method:
}

We run a Rank Ordered Logistic Regression (ROLOGIT) and calculate Disposition to Pay (WTP) for Bear Fruit Characteristic. Findings: We find that the perception of the Bear Fruits selected is contingent upon only a handful of Characteristic. We find that crispness and price are significant Characteristic in Pear. Price has a non-significant effect on preferences for grapes. Sweetness, shelf life and price significantly influence consumer choice when buying sweet oranges. Seeds that are relatively low in bitterness and softness are the two key Characteristic influencing the purchase decisions of Pomegranate.

\section{Research Limitations:}

The number of Characteristic used in the choice card was limited to most important Characteristic. Additionally, only a main effect model was estimated without considering the interaction effects of Characteristic in this study.

\section{Originality/Value:}




\section{ENGINEERING}

Favorable features of Bear Fruits in Sri Lankan condition have not been studied so far. Thus, incorporating favorable features of the Bear Fruits considered into breeding programs of the local counterparts of these Bear Fruits may be Bear Fruitful.

Keywords: Symbiotic Investigation, Consumer preference, Bear Fruit, Rank ordered log it, Disposition to pay

\section{INTRODUCTION}

Bear Fruit breeders worldwide continually attempt to create new varieties of Bear Fruit aiming at higher productivity and higher consumer acceptance. Breeding Bear Fruit varieties is a long and a time consuming process. For example, the Washington State University (WSU) breeding program for apples started in 1994 but the first release from the program was 'WA 2' variety, which was in 2009 (Evans, 2013). Thus, such innovations are a difficult and expensive process with usually low success rates. Success rates of commercial launches of innovations are about $10 \%$. Innovators see the success or failure of these breeding

Programs only at the time of commercialization when consumers decide to accept or reject the product. Therefore, Introducing new products into competitive markets need current and extensive information about consumer preferences to develop products to capture the market share. Preferences refer to consumers' expressed like or dislike for a product based on an overall evaluation or overall attitude toward the product. When consumers pay for a preferred product, one can distill the preference by splitting down products into their constituent Characteristic. For a breeder, a quantitative measurement of how each attribute function in generating overall consumer use for the product is vital to maximize consumer demand. Additionally, consumer preference information can also help firms selling products to develop specific strategies targeting niche markets and increase sales through product diversification. As in most food products, a consumer's decision to buy Bear Fruits is influenced by three 'groups' of Characteristic: search, experience and credence. Consumers ascertain search Characteristic before they purchase the Bear Fruit. Price, color, shape, are some examples. After consuming the product, they ascertain the levels of 'experience' Characteristic such as taste. Consumers can fathom some Characteristic by neither searching nor experiencing the product. This last category is the credence. Thus, third party information is necessary to the consumer to obtain values of credence Characteristic. Quality is one major credence attribute. In this research, we take on the task of assessing the relative preference for various Characteristic of four selected Bear Fruits (Dominant Bear Fruit species among urban 


\section{ENGINEERING}

consumers namely, grapes sweet orange pear and pomegranate falling into these three categories: search, experience and credence. We attempt to make quantitative measurements of consumer preference for each attribute considered.

\section{METHODOLOGY}

Conceptual framework We hypothesize that utilities that a consumer generate is as random. This means that even if a consumer prefers a certain product over others, there is a chance that the consumer to choose a less preferred product in certain instances. This selection of nonpreferred products is because of random shocks. Thus, we use the random utility framework where we assume that utility generated from choosing an alternative (a Bear Fruit profile in this study) has a latent distribution.

Assume that individual respondents rank the alternative Bear Fruit profiles. Keeping in line with the consumer behavior theory, we expect that a consumer to rank alternative $k$ over $j$ if utility from alternative $k$ is greater than the utility of alternative $j$. If we denote the rank given to each alternative as rij (the number or rank given by individual i for Bear Fruit profile j),

Symbiotic Investigation The two broad categories of non-market valuation techniques used in identifying consumer preferences are the revealed preference and the stated preference technique. The revealed preference (RP), models assume that consumers reveal their preferences by their purchasing habits. On the other hand, the stated preference (SP) technique directly asks people what they are willing to pay for a product. SP methods exist with varying names. Symbiotic Investigation (CA), Functional measurement, Tradeoff Investigation, and transfer price method are best known. For assessing consumer preference on Bear Fruit Characteristic, we use the Symbiotic Investigation (CA) technique. It is one of the most significant developments in marketing research since its introduction in 1971 by Green and Rae. This method measures the satisfaction from a product with multiple Characteristic

1990) via a systematical variety of product Characteristic by assigning to each attribute level a value that indicates the relative importance of that level to the respondents (Wang et al., 2003; Hair et al., 2010). In this study, we proceeded as given below. First, we selected Characteristic to be valued and their levels. We developed a stimulus set from the selected Characteristic. Afterwards we collected data using a pre-tested questionnaire and the stimulus and we analyzed data with a Rank Ordered Logic procedure available in Stat software. Characteristic and levels selection We conducted a literature search to understand the most important Characteristic of the selected Bear Fruits. Consumers in the northeastern US value apple 


\section{ENGINEERING}

crispness the most, followed by size, color, and flavor for apples for the fresh market. For Satsuma mandarins, seeds followed by price, color and size are important Characteristic (Benjamin et al., 2004). Simply flavor (e.g., sweetness and acidity), texture (e.g., flesh firmness and crispness), appearance and price premiums were important quality traits for the Bear Fruit crops (Gallardo et al., 2014). To verify that the Characteristic ascertained from the literature review are in line with the local preferences, we had a focus group of experts in the area, mostly consisting of the Faculty members of the university. We also interviewed consumers in deciding on the Characteristic and their levels. In an effort to refine our Characteristic and their levels further, we conducted a pilot survey with twenty respondents. We asked the respondents to rank the product profiles first and thereafter to order the Characteristic according to their importance towards them. After refining, we opted to have seven Characteristic to evaluate pomegranate and six Characteristic for pear, grapes and orange respectively. Finally, all Characteristic had two opposite levels.

Stimulus Set Construction Full factorial design of symbiotic Investigation includes all the combination of attribute levels. For example, six Characteristic with two levels for each Characteristic will come up with $2 * 2 * 2 * 2 * 2 * 2=64$ product profiles leading to a total 128 combinations for pomegranate and 64 combinations for grapes, sweet orange, and pear. Because this is not practical for a respondent to evaluate, we obtained an orthogonal fractional factorial design in SPSS (version 23) to make the task convenient by reducing profile (stimulus card) number to eight for each Bear Fruit. Partial profile is a necessity when the number of Characteristic and the levels within the Characteristic become large. It considers a subset of the entire, which would be representative of the full profile. Orthogonal process makes sure that the profiles contain the levels equally or in proportion. We applied a full profile symbiotic approach, the traditional symbiotic approach in data collection, where, we presented the subjects with stimuli that include all Characteristic and each with one of their levels. We used pictures for external Characteristic like peel color, and shape to make the exercise more realistic by providing a real market situation for the respondent. We used verbal explanation to explain other characteristics. At the bottom of the card, we left a space for ranks.

\section{Data Collection}

Our research strategy is a deductive approach using a survey. In addition to the stimulus cards to evaluate product Characteristic, we included general information to obtain respondent characteristics such as age, sex, monthly income, educational level and employment in the questionnaire administered. We conducted face-to-face interviews in three urban councils in

R.H.M. 
the Colombo district, which is the commercial hub of Sri Lanka. We obtained a systematic sample from a household list taking every fifth household into the sample and we removed households who do not consume Bear Fruits from the sample. A respondent evaluated only two Bear Fruits: either grapes and sweet orange or pear and pomegranate to reduce the effects of respondent fatigue. This resulted in two different samples for the four Bear Fruits. The respondents' task was to rate each stimulus from one to eight where one indicates most preferred and eight indicates least for the product described. We administered 120 questionnaires in each sample but had to reject some of them because they were incomplete. The final evaluation samples were 120 for pear and pomegranate and 103 for grapes and sweet oranges. We collected data during February and March in 2016. Data Investigation We carried out the data Investigation using the ROLOGIT procedure available in Stata (Version 14). For the ease of interpretation, we calculated odds ratios by exponentiation of the model's coefficients (Long and Freese, 2006). The relative importance of Bear Fruit Characteristic are a vital piece of information for breeders, thus, we calculated the Disposition to Pay (WTP) for each attribute enabling comparison of Characteristic. Equation (1) assumed that utility is composed of a systematic component ( $\mu \mathrm{ij})$ and a random component. We assumed that the random component has the following additive; linear relationship with Bear Fruit Characteristic (z) and price of the Bear Fruit (p); $\mu \mathrm{ij}=\gamma_{\mathrm{zj}}+\alpha \mathrm{pj}(5)$

By differentiating equation (5) with respect to each attribute ( $\mathrm{j}$ ij z $\partial \partial \mu$ ), we obtained the marginal utility provided by the attribute. By differentiating with respect to price ( $\mathrm{j}$ ij $\mathrm{p} \partial \partial \mu$ ), we got the marginal utility of price. The WTP or the marginal rate of substitution between attribute zj and money is the ratio between these two derivatives.

\section{RESULTS AND DISCUSSION}

Descriptive Statistics of the Samples Both the samples used for the study are not considerably different from each other. Sample focusing on pear and pomegranate has79 females and 23 males. Descriptive statistics showed that 45 percent of them are educated up to their tertiary education level, 28 percent obtained higher education and only three percent of them had only primary education. More than $3 / 5$ of the respondents in the sample have employment. The second sample is also female dominated ( $>70$ percentage). Forty three percent of respondents have education up to tertiary level and 40 present covered their tertiary education. Eighteen percent obtained secondary education and only four percent up to primary education. There were 56 employed respondents and 47 reported as non-employees. The participants' ages ranged from 16 to 72 years. In both the samples, mean age was around 41 years and income is

R.H.M. 
around Rs.50, 000. Results of Rank Ordered Logistic Regression The results of the ROLOGIT are in Table 2. We report coefficients, $p$-values and the Disposition to Pay (WTP) values for each attribute in the four Bear Fruits. For easy comparison.

In pears, we considered six Characteristic including price. We find that only crispness and price are significant. From the calculated WTP values, crispness show the highest WTP among all Characteristic showing its importance relative to others (Table 02 and Figure 03). Accordingly, consumers are willing to pay Rs.3 more for 'crisp' pears. Crispiness of pears is a maturity indicator (Manning, 2009). Previous literature also reports consumer preferences for crispy pears (Hoehn et al., 1996). We find that three Characteristic return negative values: peel color, size and sweetness. Pear comes in two colors: green and yellow. But the results tell us that consumers value yellow peel color over green. At present, the Sri Lankan Department of Agriculture is conducting research to introduce Asian pear (Pyrus pyrifolia) in the country because it is more suited for the climate than the previously introduced European varieties. These carry a yellow/brownish color peel and consumers may prefer these over the green ones. Contradictory to expectation, the size variable gave a negative sign meaning that consumer preference for smaller pears over larger ones. Also, the results suggest that consumers like less sweet pears than sweeter varieties. Results also show that consumers are interested in the shape of the Bear Fruit as well. However, the critical attribute in pears is the crispness. In grapes all Characteristic are significant except price. Nevertheless, we calculate the WTP values in order to compare Characteristic. We see two Characteristic with negative WTP values: presence of seeds and firmness. Surprisingly, consumers prefer seeded grapes. They are willing to pay about Rs1.51 to avoid seedless grapes.However, seeded grapes may have a slight nutritional benefit over seedless grapes due to the additional protein, minerals and fats (including omega-3s) that are available in the edible seeds (Russell, 2009). This may be the reason to prefer seeded grapes. Further, they are willing to pay about Rs 2 to avoid soft Bear Fruits. Firm Bear Fruits are prefered. On the otherhand, consumers would be willing to pay Rs.9.36 for safety assured grapes. This is the highest Disposition to pay for any trait with respect to

grapes. However, safety cannot be searched or experienced unless provided by a third party to the consumer. Piva et al. (2006) also reported that demand for grapes in Spanish markets can be improved by using quality marks. Further, consumers are willing to pay for sweetness than the size of the Bear Fruit. Therefore, based on the WTP values, consumers consider safety, 


\section{THE AMERICAN JOURNAL OF}

AGRICULTURE AND BIOMEDICAL

VOLUME01 ISSUE01

ENGINEERING

sweetness, firmness, absence of seeds and shape respectively in their buying decisions of grapes.

\section{CONCLUSIONS}

This study investigated the consumer preference for Bear Fruit Characteristic using a symbiotic Investigation. Results show that there are key Characteristic that consumers are concerned on when they are purchasing Bear Fruits. Crispness is the critical attribute in pear while bitterness and hardness of seeds in pomegranate. Consumers look for food safety in grapes and shelf life in sweet orange. Sri Lanka imports most of these Bear Fruits and the country has a substitute for each of these Bear Fruits. There is a 'general' preference for these imported varieties of Bear Fruits by consumers. However, for developing nations such as Sri Lanka, import substitution of these Bear Fruits may save a significant amount of foreign exchange while giving employment opportunities to the rural poor where agriculture is the mainstay.

Thus, by incorporating these preferred Characteristic into local varieties may prove Bear Fruitful.

\section{REFERENCES}

Allison, P. D. and Christakis, N. A. (1994). Logit models for sets of ranked items. Sociological methodology. 24: 199-228. DOI: https://doi.org/10.2307/270983 Beggs, S., Cardell, S. and Hausman, J. (1981). Assessing the potential demand for electric cars. Journal of econometrics. 17(1): 1-19. DOI: http://dx.doi.org/10.1016/0304-4076(81)900567 Breidert, C., Hahsler, M. and Reutterer, T. (2006). A review of methods for measuring Dispositionto-pay. Innovative Marketing. 2(4): 8-32. https://michael.hahsler.net/research/misc/ InnovativeMarketing_2006.pdf Chapman, R. and Staelin, R. (1982). Exploiting Rank Ordered Choice Set Data Within the Stochastic Utility Model. Journal of Marketing Research 19: 288301. DOI: http://dx.doi. org/10.2307/3151563 